\title{
Spatial mapping of macrophage heterogeneity in murine atherosclerotic plaques
}

\section{Pieter Goossens $^{1}$, Chang Lu ${ }^{1}$, Marc van Zandvoort ${ }^{2}$, Marjo Donners ${ }^{1}$, Marion Gijbels ${ }^{1}$, Erik Biessen ${ }^{1}$}

\section{Introduction}

\begin{abstract}
Atherosclerosis-related CVDs are the leading causes of death worldwide. As the most abundant immune cell type in plaques, macrophages play crucial roles in the atherosclerotic pathogenesis. Due to their versatile and plastic phenotype in response to locally-produced factors, they are very heterogeneous and can exert both pro- and anti-atherogenic activities. As context, and hence macrophage heterogeneity, is highly disease stage-dependent, this considerably complicates the design of effective macrophage-targeting therapies. To fully dissect their impact and the different subsets individual roles in the pathogenesis, a complete and comprehensive mapping of the intraplaque myeloid phenotypic landscape would be necessary.

In recent publications, newly-emerging approaches such as mass cytometry and single-cell RNAseq underpinned this heterogeneity, but interpreting the classification's functional implications remains difficult since both techniques suffer from the same caveats: not only is all spatial information on the cells' location lost during tissue disruption steps to obtain single-cell suspensions, specific subsets (e.g. the fragile foam cells) will also be lost during this procedure, leading to an underestimation of their contribution to the disease progress.

In immunohistochemistry (1-2 colors) or immunofluorescence (3-5 colors) on the other hand, no cells are lost and all information on their location is retained, but these methods lack the high phenotypic resolution required to distinguish the differen subsets. We therefore developed a multispectral imaging approach.
\end{abstract}

\section{Multispectral imaging}
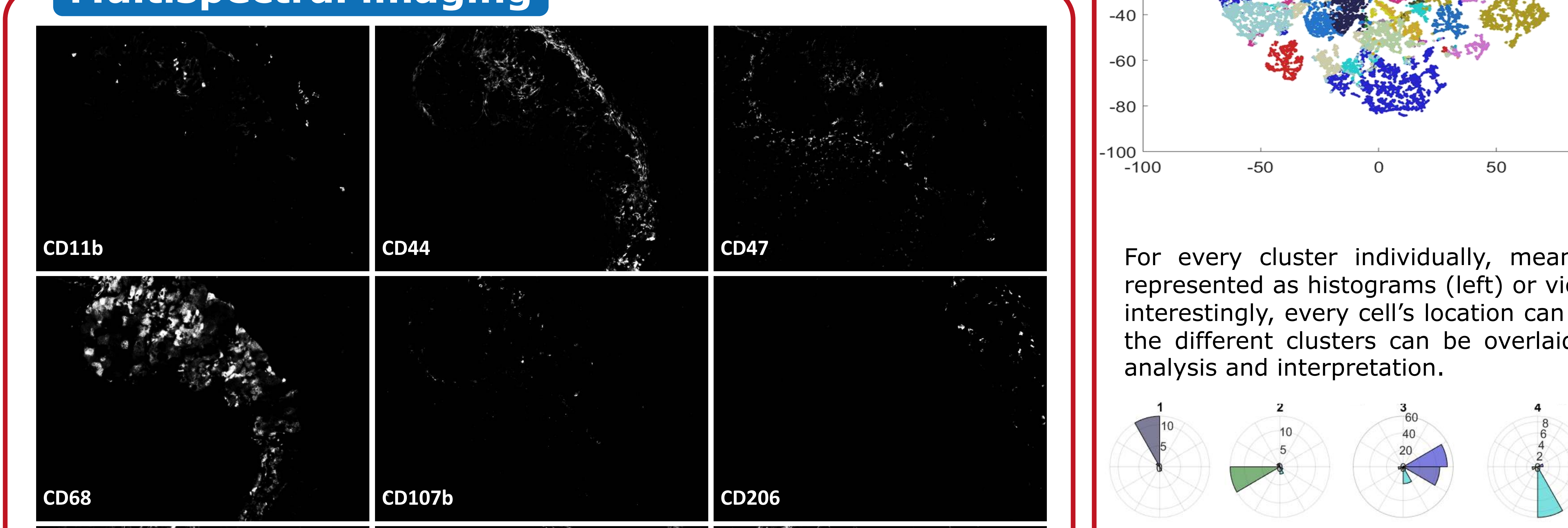

\section{Visualization \& analysis}

A $k$-medoids clustering based on cosine distance identified the 30 clusters of cells represented in the tsne plot (left). Visualization of these clusters in a $k$-nearest neighbor network (top, $k=3$ ) or a minimal spanning tree (bottom, cluster identifier in red and number of cells in black) reveals how a few distinct subsets can be further subdivided into more subtly differing cluster members that form a continuum between the most extreme phenotypes (foam cells vs. inflammatory macrophages).
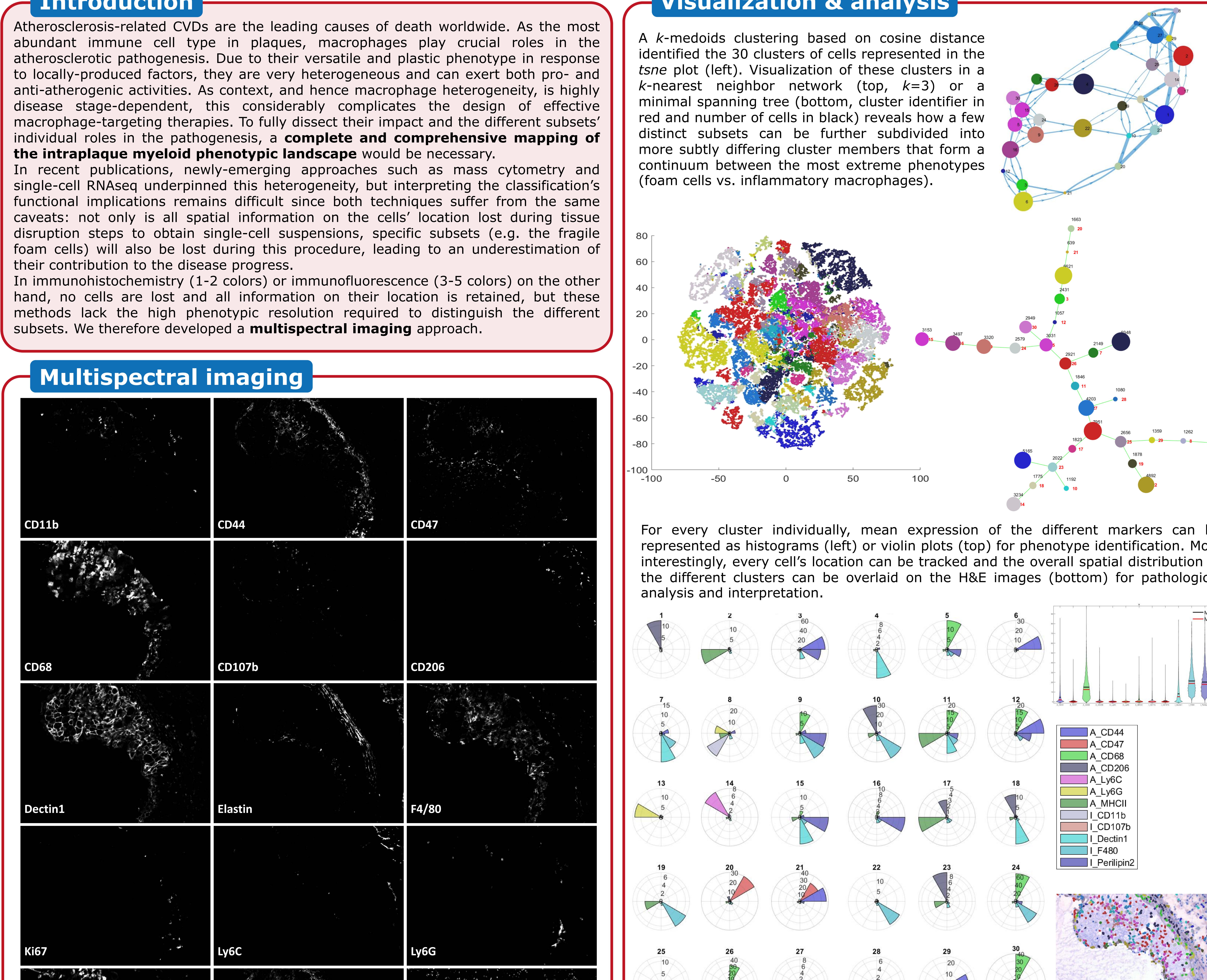

CD47
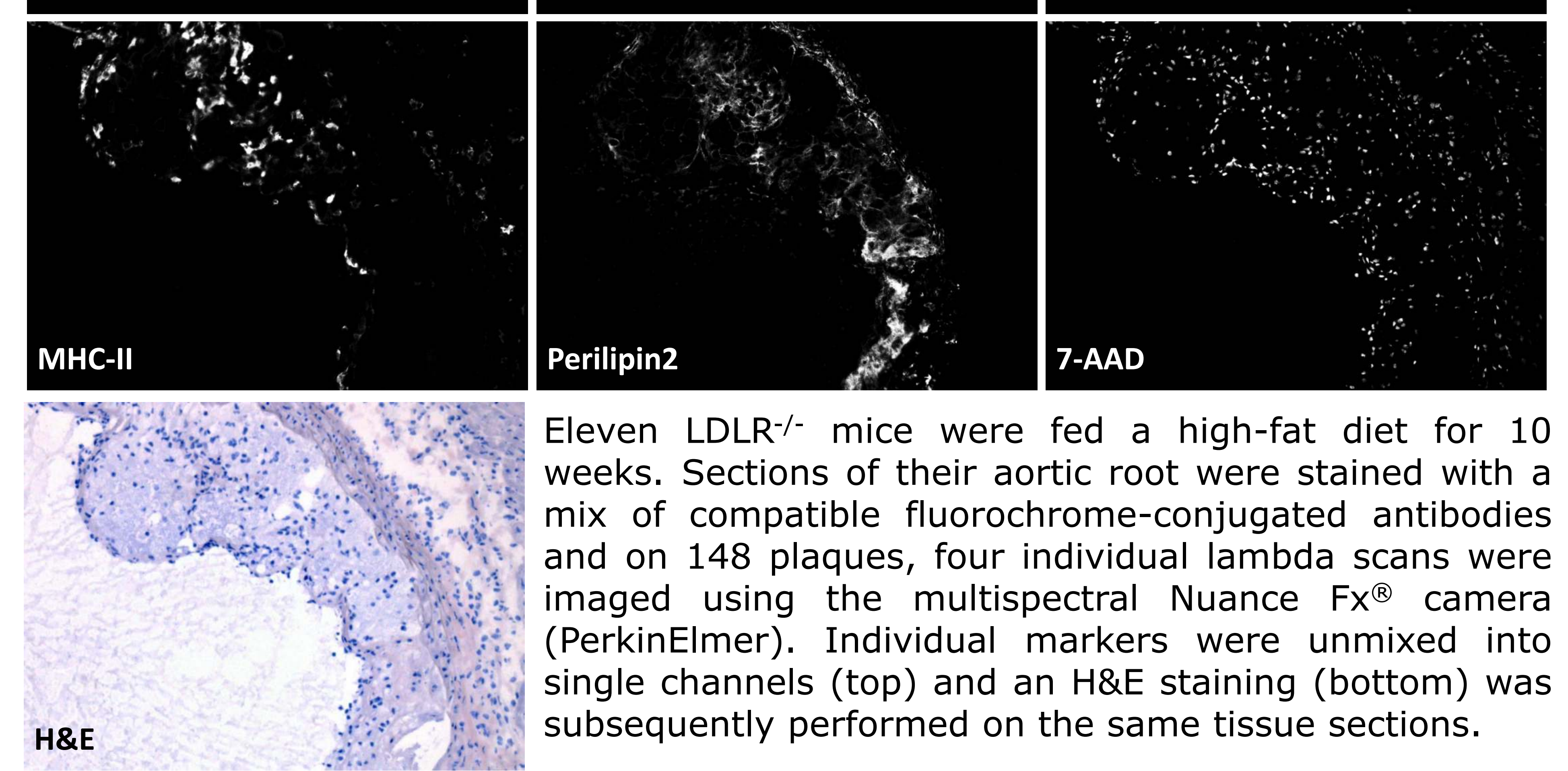

H\&E

\section{Data processing}

Overlaying a cell segmentation mask on the 12 phenotype-defining fluorescent channels (all but elastin, Ki67 and 7-AAD) resulted in a matrix of mean fluorescent intensities of these markers for each individual cell, which was normalized and compensated for spillover from neighboring cells. After leaving out the cells negative for all markers, different approaches were compared to cluster the remaining 88,495 cells based on their similarity in marker expression pattern (SOM, FlowSOM, Xshift, hierarchical clustering, $k$-means, $k$-medoids all with different numbers of clusters) and their accuracy was compared in silhouette plots (right).

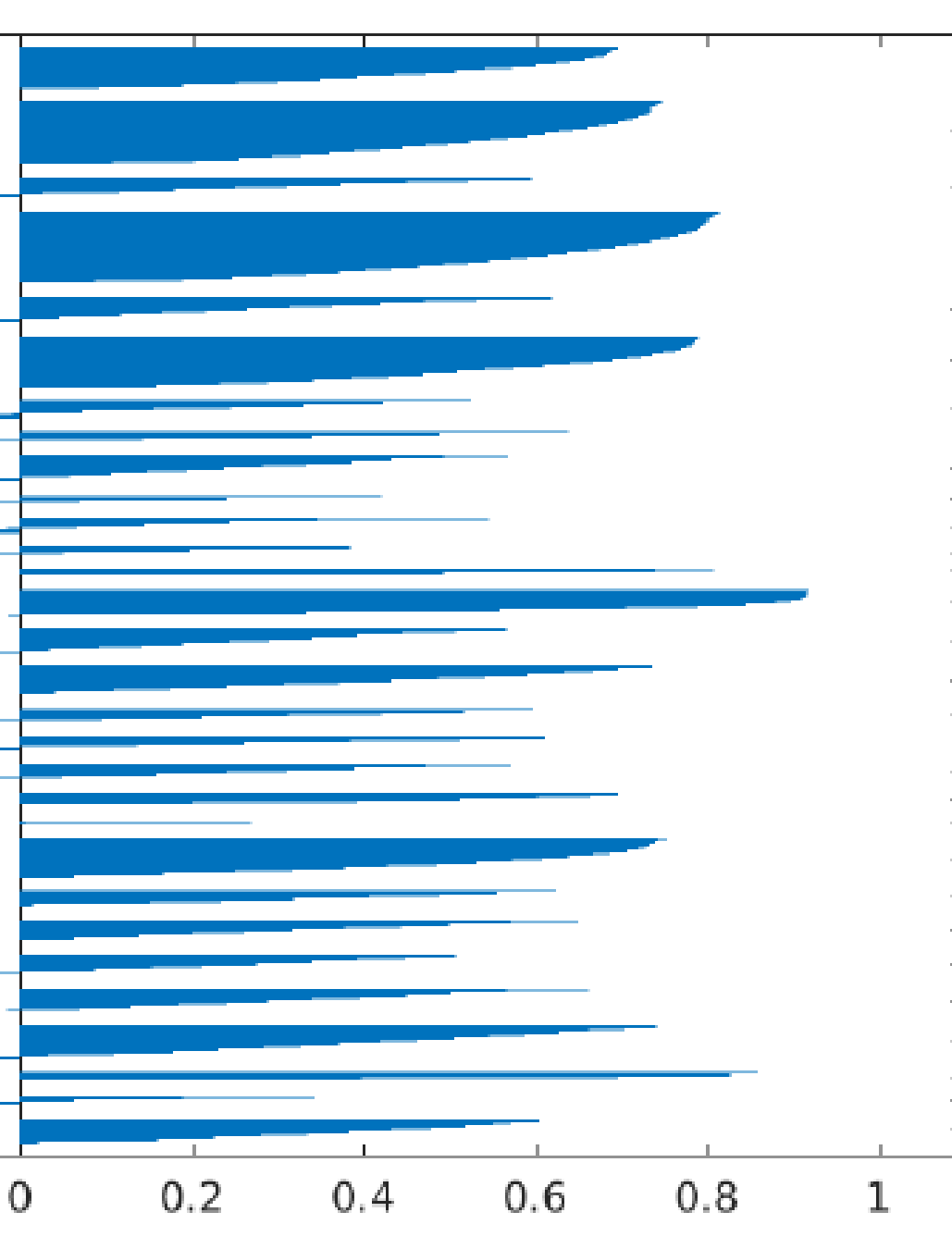

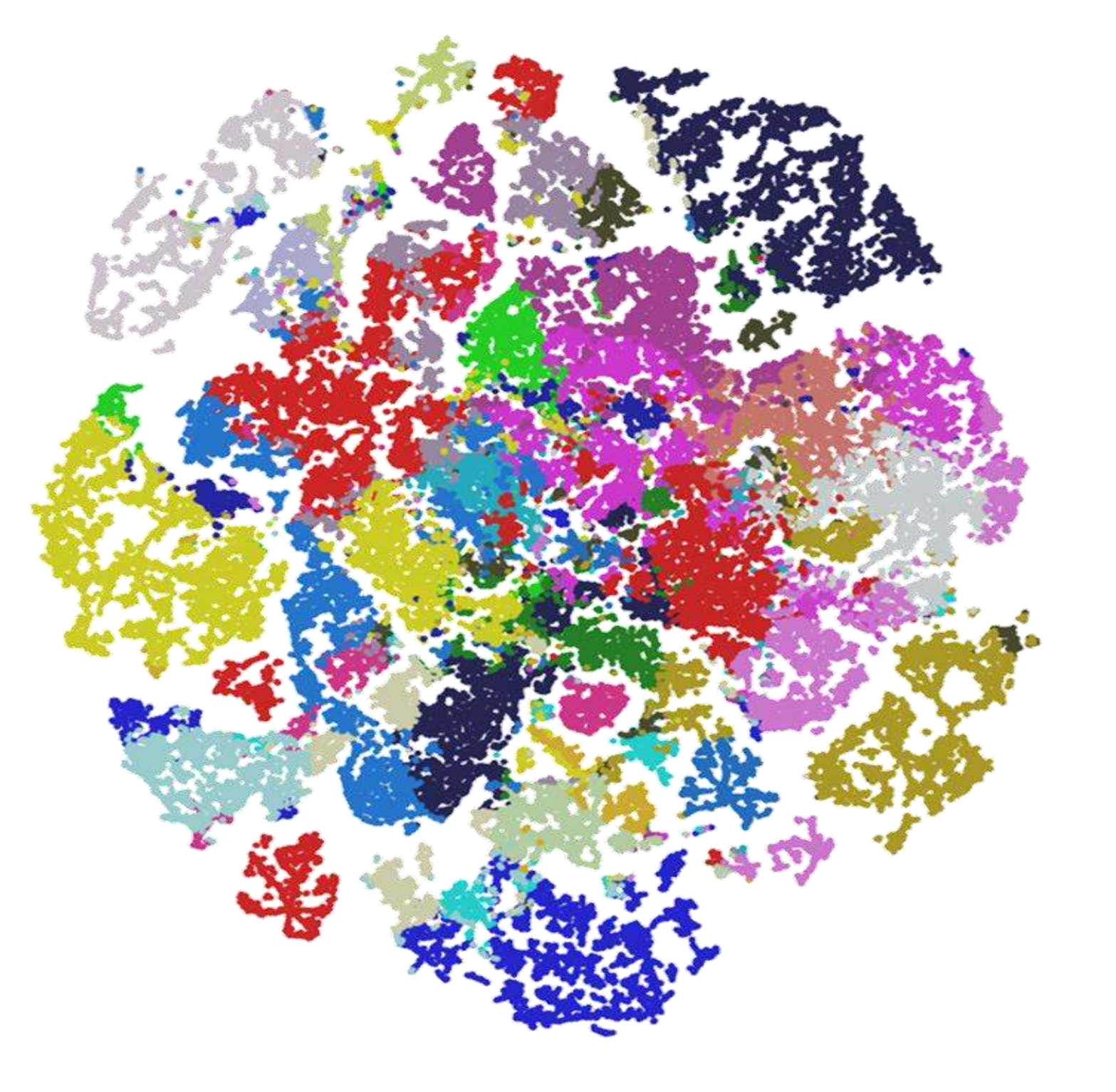

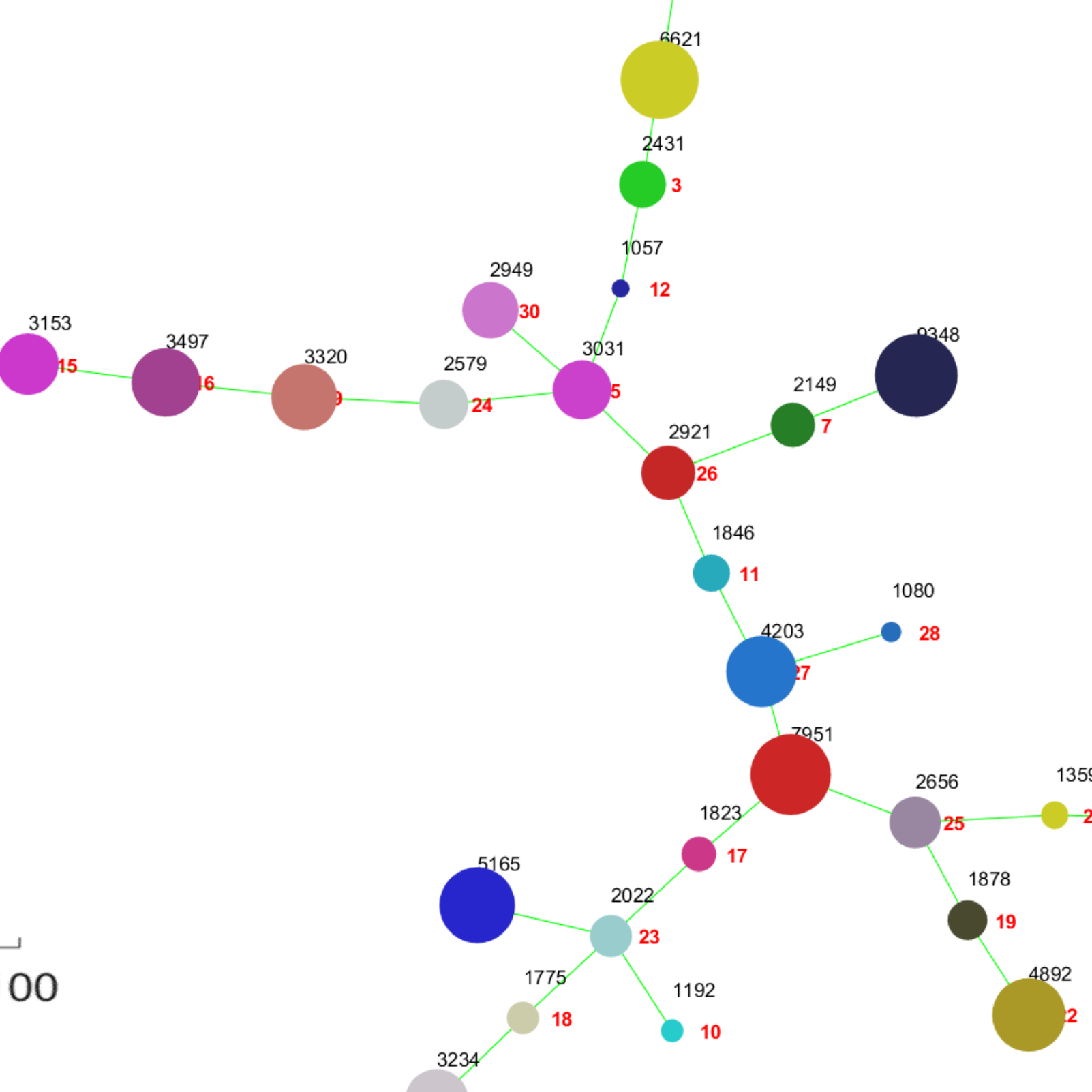

For every cluster individually, mean expression of the different markers can be represented as histograms (left) or violin plots (top) for phenotype identification. Most interestingly, every cell's location can be tracked and the overall spatial distribution of the different clusters can be overlaid on the H\&E images (bottom) for pathological analysis and interpretation.

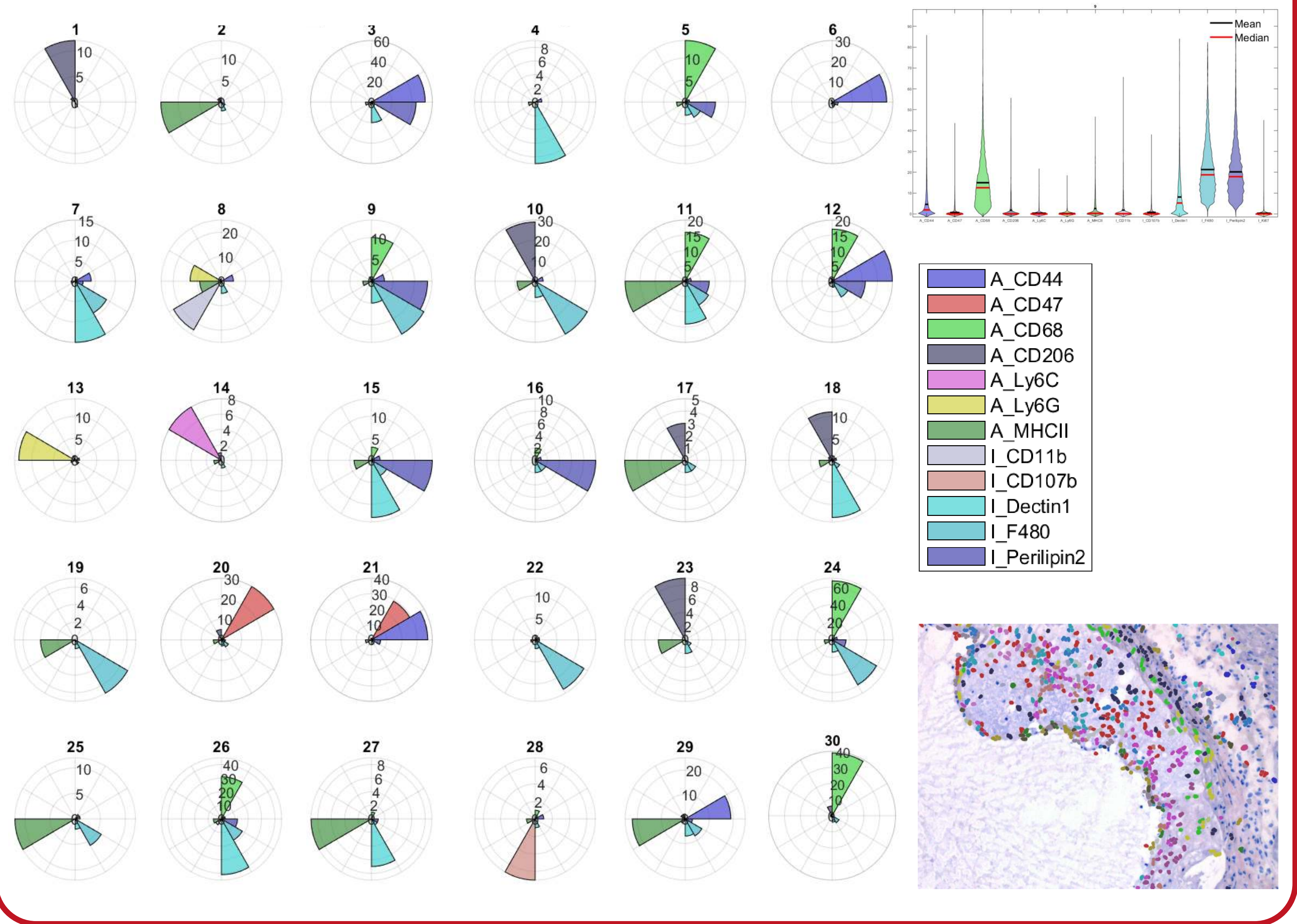

Advantages over single-cell approaches

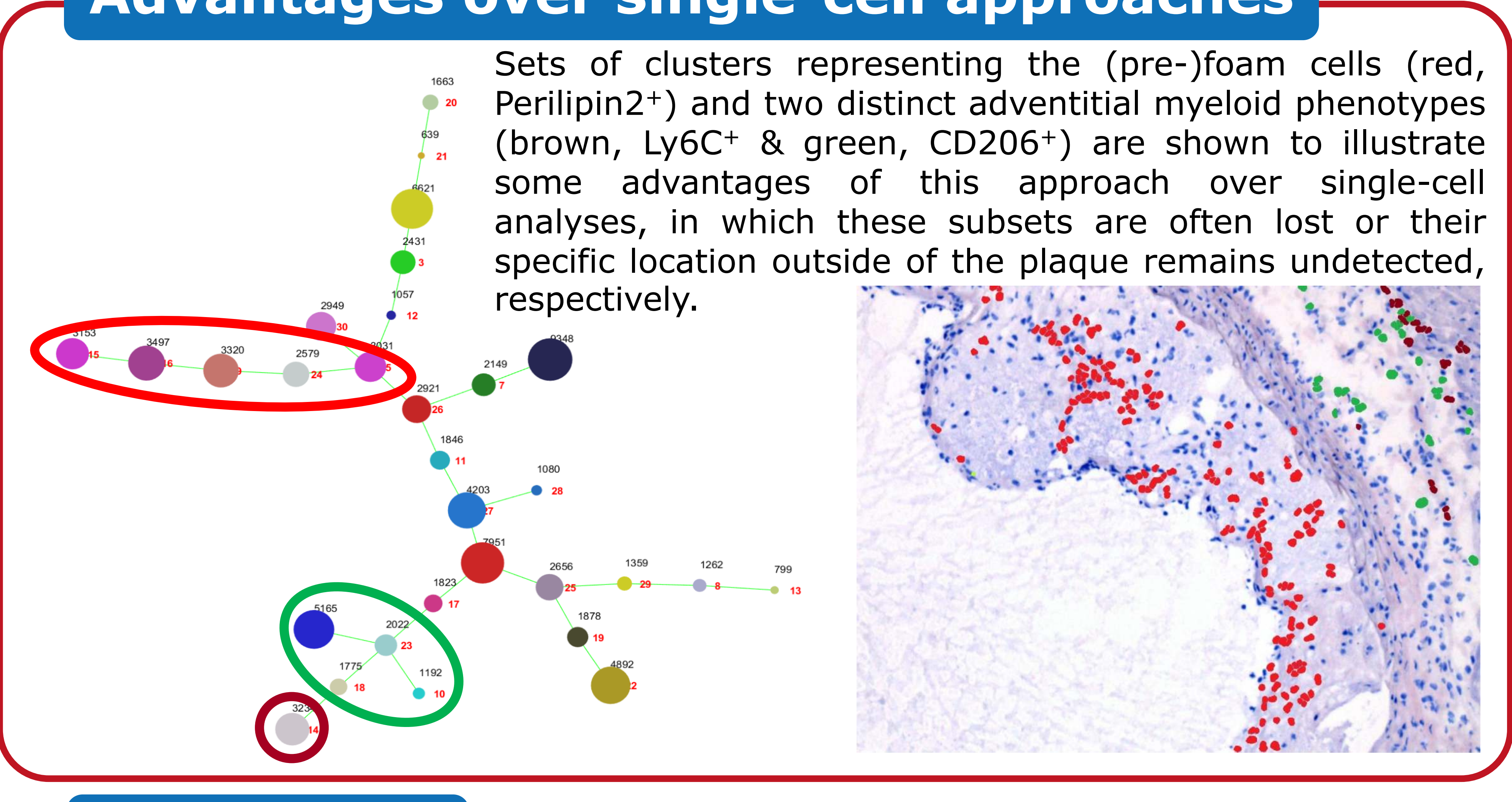

\section{Conclusions}

This multispectral imaging approach on murine plaques resulted in the characterization of plaque myeloid heterogeneity with a phenotypical resolution comparable to cytometry but avoiding the cell detection bias induced during tissue processing and retaining all spatial information on each individual cell. Following up on this, further analyses can be envisioned, such as quantification of cell co-localization with other cell types (cell communities) or with plaque domains (niches), or characterization of the (molecular and/or metabolic) micro-environment in adjacent tissue sections, shedding light on their influence on the plaque macrophages' phenotypes and functions. 